\title{
Photospheric modeling through spectral line inversion
}

\section{Temperature and radial velocity stratifications and fluctuations}

\author{
J. Koza ${ }^{1,2}$, A. Kučera ${ }^{2}$, J. Rybák², and H. Wöhl ${ }^{3}$ \\ 1 Sterrekundig Instituut, Universiteit Utrecht, PO Box 80000, 3508 TA Utrecht, The Netherlands \\ e-mail: koza@astro.sk \\ 2 Astronomical Institute, Slovak Academy of Sciences, 05960 Tatranská Lomnica, Slovakia \\ 3 Kiepenheuer-Institut für Sonnenphysik, Schöneckstrasse 6, 79104 Freiburg, Germany
}

Received 23 May 2006 / Accepted 6 July 2006

\section{ABSTRACT}

\begin{abstract}
Aims. We aim to determine average radial stratifications of various physical parameters throughout the solar photosphere at high angular resolution for non-magnetic and magnetic areas and to compare these with standard semiempirical 1D modeling and with 3D hydrodynamics (HD) and magnetohydrodynamics (MHD) simulations.

Methods. We analyse a 15-min sequence of adaptive-optics spectrograms of very high angular resolution taken at solar disk centre. We split the data between a quiet area and a magnetic one and derive mean temperature and velocity stratifications and fluctuations for these separately by applying LTE inversion based on response functions.

Results. The mean temperature stratifications in the non-magnetic region agree well with the classical 1D models and the 3D simulations at all heights. However, the observed rms temperature is much lower than in the simulations, the observed mean velocities indicate more upflows, and the observed velocity fluctuations are smaller except in upper layers. Some of the discrepancies are likely to result from remaining smearing by atmospheric seeing and instrumental limitations. The magnetic area shows conspicuous behaviour at large height. We also find evidence of fast low-photosphere downflows in the magnetic area and of enhanced temperature above a small pore.
\end{abstract}

Key words. Sun: photosphere - Sun: granulation - Sun: oscillations - stars: atmospheres

\section{Introduction}

Solar high-resolution spectroscopy provides a framework for quantitative comparison of actual photospheric properties with classical semiempirical 1D models of the quiet solar photosphere and with newer 3D radiation-hydrodynamics simulations of the solar granulation. In this paper, we analyse new high-resolution spectrocopic data in this context. We apply an inversion method to a 15-min time sequence of high-resolution spectrograms to obtain the space-time evolution and the vertical stratification of different physical parameters throughout the solar photosphere (cf. Puschmann et al. 2003, 2005). In this paper we concentrate on temperature fluctuations, line-of-sight velocities, and the macroturbulent velocity.

Solar 3D hydrodynamics simulations such, as the one by Stein \& Nordlund (1998), were recently extended to the low chromosphere by Wedemeyer et al. (2004) and to the realm of magneto-convection by Vögler et al. (2005). These 3D simulations all assume strict LTE but differ in their treatment of opacity and in how they use the opacity packages. In this paper, we use the inversion code SIR by Ruiz Cobo \& del Toro Iniesta (1992) as a powerful tool for filling the gap between these 3D numerical simulations of the solar photosphere and the older semiempirical $1 \mathrm{D}$ modeling. We aim to add verification of the 3D simulations to their comparison with various observations summarised in Stein \& Nordlund (2000). We do this by comparing the results of our data inversions to the temporally and spatially-averaged simulation stratifications tabulated in Asplund et al. (2004, 2005). The pertinent results are the mean temperature stratifications, rms fluctuations, and the relations between the continuum intensity and the velocity. In our comparisons, we distinguish between a non-magnetic region and a small region with enhanced magnetic activity. We also investigate whether the magnetic field in a pore is the single one responsible for the observed increase in the full width at half minimum $(F W H M)$ and in line-centre intensities. This provides further evidence of enhanced temperatures above small pores as reported by Sütterlin et al. (1996) and Sütterlin (1998) and predicted by the simulation of Stein \& Nordlund (2000). This work goes well beyond our previous attempts aimed at temporal evolution of physical parameters within a single granule and intergranular lane (see Kučera et al. 2004, and references therein) and continues the quantitative confrontation of observations with simulations.

\section{Observations and data reduction}

We use a time sequence of spectrograms obtained at the German Vacuum Tower Telescope (VTT, Schröter et al. 1985) at the Observatorio del Teide on April 28, 2000. The adaptive optics of the National Solar Observatory at Sacramento Peak (Rimmele et al. 1999) was operated in observations. The spectrograms around $4993.8 \AA$ and $6456.5 \AA$ (Fig. 1) were taken synchronously at a 3 -s cadence with an exposure time of $1 \mathrm{~s}$, and the observing run lasted for $15 \mathrm{~min}$ from 9:26:39 UT to $9: 41: 33$ UT. The width of the slit was $100 \mu \mathrm{m}$ equivalent to 0.46 . The spectral data were acquired with two $2 \mathrm{k} \times 2 \mathrm{k}$ 12 bit XEDAR XS-2048-A-12 CCD cameras using $2 \times 2$ pixel 

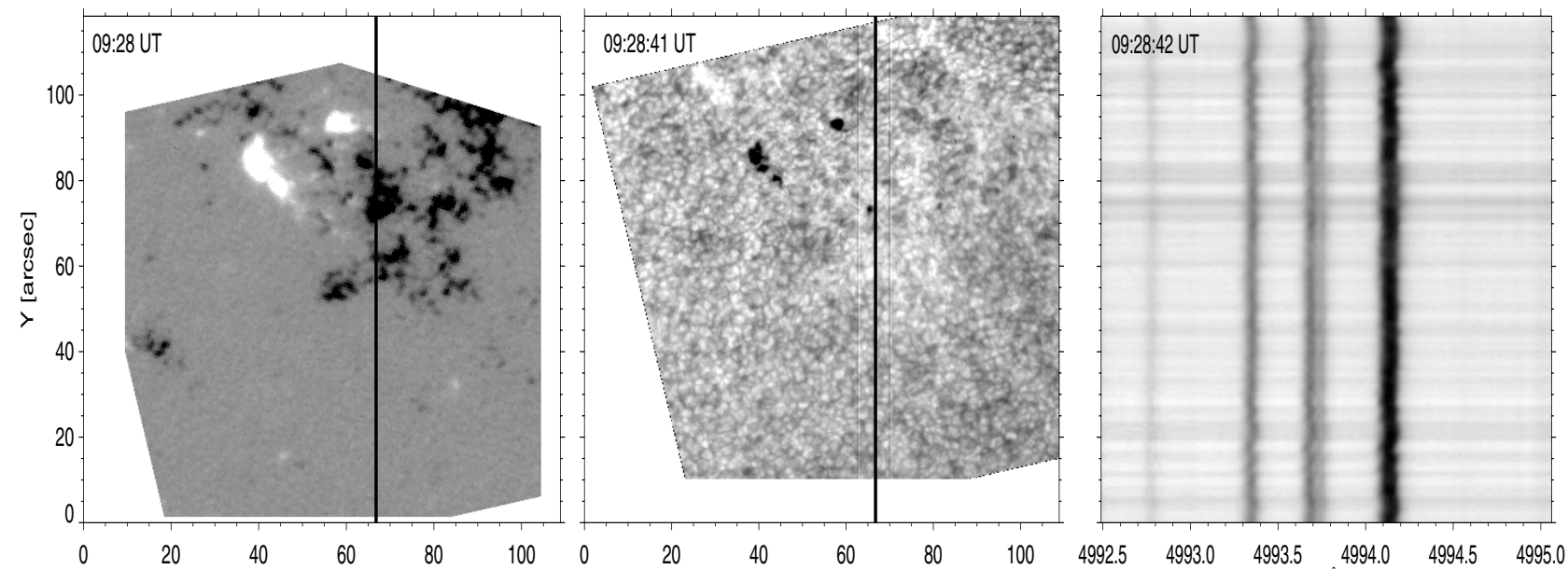

Fig. 1. Left: MDI/SoHO magnetogram. Middle: G-band slit-jaw filtergram. Right: example spectrogram. The slit position is indicated by the vertical black line. In the magnetogram the completely black and white regions involve $\pm 300 \mathrm{G}$ and higher field strengths. The regions at $y=0-55^{\prime \prime}$ and $y=60-95^{\prime \prime}$ (measured along the slit) are indicated as non-magnetic and magnetic, respectively.

hardware binning, doubling the linear dimension of an individual pixel to $28 \mu \mathrm{m}$. The spatial sampling was $0.126^{\prime \prime} / \mathrm{pixel}$, and the dispersion was $2.528 \mathrm{~m} \AA /$ pixel and $3.066 \mathrm{~m} \AA /$ pixel for the first and second spectral regions, respectively. The corresponding Doppler velocities per pixel were $142 \mathrm{~m} \mathrm{~s}^{-1}$ and $152 \mathrm{~m} \mathrm{~s}^{-1}$. In particular, we used the G-band filter of $9 \AA$ bandpass centred at $4306 \AA$ for the slit-jaw image acquisition. For more details, see Rybák et al. (2004) and Odert et al. (2005).

The entrance slit of the spectrograph was positioned almost in the centre of the solar disk $(\mu \approx 0.96)$ near the AR 8976, partly on a quiet photosphere ranging from $0^{\prime \prime}$ to $55^{\prime \prime}$ (Fig. 1) and partly on a small unipolar region of enhanced magnetic activity ranging from $60^{\prime \prime}$ to $95^{\prime \prime}$. Hereafter, these regions are referred to as non-magnetic and magnetic, respectively. Inspecting the MDI/SoHO magnetogram and the G-band slit-jaw filtergram (Fig. 1) of the magnetic region we conclude that the region involved anomalous granulation and many G-band bright points harboring the flux tubes, so the region would reveal itself as a plage in the chromosphere and the faculae was its limb counterpart. Moreover, the spectrograph slit grazed a pore situated at $y=73^{\prime \prime}$ whose longitudinal component of the magnetic field strength was approximatively $-900 \mathrm{G}$. Another dark band is also conspicuous between $y=80^{\prime \prime}$ and $84^{\prime \prime}$ at the spectrogram and the map of continuum intensity (Figs. 1 and 6). We suspect that the small micropore and the area of higher concentration of intergranular lanes visible in the G-band filtergram at the positions $y=80-84^{\prime \prime}$ are responsible for this feature. The shift in the micropore with respect to the slit apparent on this particular G-band filtergram was probably caused by the seeing. The estimated diameters of the pores are not larger than 2-3".

Generally, the seeing conditions were of good quality allowing for sub-arcsecond resolution. The quality of the spectra, indicated by the relative contrast of the continuum intensity $\delta I_{\mathrm{rms}}$ is displayed in Fig. 2. The mean $\delta I_{\mathrm{rms}}$ measured in the non-magnetic region are $3.4 \%$ and $2.5 \%$ in the spectral regions $4993.8 \AA$ and $6456.5 \AA$, respectively.

In the process of data reduction no correction for the 5-min oscillations was performed. After the various reduction steps (Wöhl et al. 2002), the spatial dimension of the spectra resulted in the value of 1008 pixels (127").

The orientation of the slit perpendicular to the horizon allowed compensation for the differential refraction. The value of

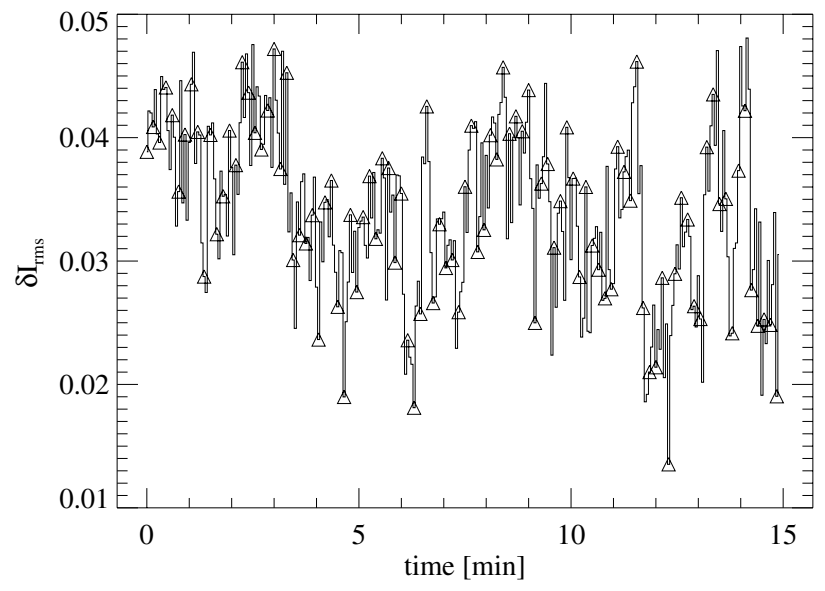

Fig. 2. Variations in the rms of the continuum intensity in the nonmagnetic region. The triangles mark the records selected for inversion.

the displacement was determined empirically by correlation of the intensities of the continua in each pair of spectra recorded synchronously by the cameras. The variation of differential refraction in the range of values from 3 pixels to 4 pixels, i.e. from $0 . ' 4$ to 0 ' 5 was found, in perfect agreement with the theoretical value of 0.5 when actual air column mass and the geographical location are taken into account. To compensate for this displacement, the spectra were shifted parallel to the slit for quoted values. After the compensation of the differential refraction the spatio-temporal extent of the investigated data volume was $118^{\prime \prime} \times 15 \mathrm{~min}$.

The wavelength calibration of the spectrograms was carried out for the mean profiles by applying the method outlined in Allende Prieto \& García López (1998a), which proved that the FTS solar atlas (Neckel 1999) can be used as a benchmark to perform wavelength calibrations of astronomical spectra and also to set up an accurate wavelength scale for solar spectra. The standard deviations of the used wavelength standards were $\approx 2 \mathrm{~m} \AA$ (Allende Prieto \& García López 1998b) corresponding to an uncertainty of $90 \mathrm{~m} \mathrm{~s}^{-1}$ in the inferred line-of-sight velocities. By applying this wavelength calibration method, we also compensated for the velocity of $90 \mathrm{~m} \mathrm{~s}^{-1}$ of the spectrograph toward the Sun caused by the orbital and rotation velocities of the Earth. 
Table 1. Parameters of the spectral lines.

\begin{tabular}{llccccccccccc}
\hline \hline Element & $\begin{array}{c}\lambda_{\text {lab }} \\
{[\AA]}\end{array}$ & $\begin{array}{c}W_{\lambda} \\
{[\mathrm{m} \AA]}\end{array}$ & Multiplet No. & $\begin{array}{c}h \\
{[\mathrm{~km}]}\end{array}$ & $\log \tau_{5}$ & $g_{\text {eff }}$ & $\begin{array}{c}\mathrm{EP}_{\text {low }} \\
{[\mathrm{eV}]}\end{array}$ & $\log (g f)$ & Term designation & $\begin{array}{c}\alpha \\
{\left[a_{0}^{2}\right]}\end{array}$ \\
\hline $\mathrm{Fe}$ I & 4992.7804 & 8 & 1110 & 134 & -1.0 & 1.3 & $(0.75)$ & 4.260 & -2.270 & $\mathrm{z}^{3} \mathrm{P}_{1}^{\mathrm{o}}-\mathrm{g}^{5} \mathrm{~F}_{1}$ & 0.281 & 993 \\
$\mathrm{Fe}$ II & 4993.3505 & 34 & 36 & 169 & -1.2 & 0.6 & $(0.7)$ & 2.807 & -3.730 & $\mathrm{~b}^{4} \mathrm{~F}_{4 \frac{1}{2}}-\mathrm{z}^{6} \mathrm{P}_{3 \frac{1}{2}}^{\mathrm{o}}$ & - & - \\
$\mathrm{Fe}$ I & 4994.1295 & 95 & 16 & 597 & -4.7 & 1.5 & $(1.5)$ & 0.915 & -2.956 & $\mathrm{a}^{5} \mathrm{~F}_{4}-\mathrm{z}^{5} \mathrm{~F}_{3}^{\mathrm{o}}$ & 0.245 & 246 \\
$\mathrm{Ca}_{\mathrm{I}}$ & 6455.598 & 48 & 19 & 246 & -1.7 & 1.0 & $(1.1)$ & 2.523 & -1.370 & $3 \mathrm{~d}_{4} \mathrm{~s}^{3} \mathrm{D}_{2}-3 \mathrm{~d}_{4} \mathrm{p}^{1} \mathrm{D}_{2}^{\mathrm{o}}$ & 0.241 & 365 \\
$\mathrm{Fe}$ II & 6456.383 & 57 & 74 & 270 & -1.9 & 1.2 & $(1.2)$ & 3.903 & -2.082 & $\mathrm{~b}^{4} \mathrm{D}_{3 \frac{1}{2}}-\mathrm{z}^{4} \mathrm{P}_{2 \frac{1}{2}}^{\mathrm{o}}$ & - & - \\
\hline
\end{tabular}

To limit the considerable processing, we performed several reduction steps decreasing the volume of the original data but keeping sufficiently high spatial, temporal, and spectral resolution. Thus the final dimension of the data cube entering the inversion was 471 spectral rows $\times 448$ spectral points $\times$ 100 spectrograms.

\section{Inversion procedure}

The inversion code SIR (Ruiz Cobo \& del Toro Iniesta 1992) used in this study consists in two basic modules. The radiative transfer module assumes LTE, homogeneous plane-parallel layers, and hydrostatic equilibrium. The inversion module implements a nonlinear least-square Marquardt algorithm (Press et al. 1992) minimizing the misfit between observed and synthetic spectral line profiles by iterative modifications for the starting model of the solar photosphere. As a result of the inversion, SIR returns the stratification of temperature, electron pressure, lineof-sight velocity, microturbulent velocity, total gas pressure, and density throughout the atmosphere. The resulting physical parameters are given both on an optical depth scale $\log \tau_{5}$ at $\lambda=$ $500 \mathrm{~nm}$ and as a function of geometrical height assuming hydrostatic equilibrium. The code also provides a macroturbulent velocity, which is assumed to be independent of depth. The chosen starting model Grevesse \& Sauval (1999) (Fig. 4) was discretised in equally-spaced logarithmic grid of the optical depth scale from $\log \tau_{5}=0.8$ to $\log \tau_{5}=-4.0$ (step size $\Delta \log \tau_{5}=0.1$ and 49 depth points). The microturbulent velocity $v_{\text {mic }}$, the lineof-sight velocity $v_{\text {los }}$ and the magnetic field strength $B$ were initialised to the depth-independent values of $800 \mathrm{~m} \mathrm{~s}^{-1}, 636 \mathrm{~m} \mathrm{~s}^{-1}$ and $0 \mathrm{G}$, respectively, at all depth points. For the macroturbulent velocity $v_{\text {mac }}$, the starting value of $1.6 \mathrm{~km} \mathrm{~s}^{-1}$ was adopted. Once the starting model was specified, the SIR code also needed to prescribe the number of equidistantly distributed nodes in which the code is allowed to modify the starting model. In this analysis, the temperature and line-of-sight velocity were perturbed subsequently in one, three, and five nodes. One node was assigned to both micro- and macroturbulent velocity throughout the inversion, whereas the initial $B=0 \mathrm{G}$ was kept unchanged.

Table 1 lists selected parameters of the five spectral lines used in the inversion. The sources of laboratory central wavelength $\lambda_{\text {lab }}$ are Nave et al. (1994) for the Fe I lines, Kupka et al. (1999) (VALD database) for the Ca I and Fe II $6456.4 \AA$ lines, and Borrero \& Bellot Rubio (2002) for the Fe II $4993.3 \AA$ line. The equivalent widths $\mathrm{W}_{\lambda}$ and multiplet numbers were taken from Moore et al. (1966). The formation heights of the line cores $h$ were taken from Gurtovenko \& Kostyk (1989) and Sheminova (1998) for the strong line Fe I 4994.1 $\AA$. The optical depth of formation of the line cores $\log \tau_{5}$ corresponds to the optical depth in continuum at $\lambda=500 \mathrm{~nm}$ in the model atmosphere HOLMU (Holweger \& Müller 1974). The effective Landé factors $g_{\text {eff }}$ were taken from VALD and the values in parenthesis were computed according to Beckers (1969). The sources of the excitation potential of the lower level $\mathrm{EP}_{\text {low }}$ are Nave et al. (1994) for the Fe I lines and the database VALD for Fe II and Ca I lines. The term designations were taken from Moore (1972) and the values of the velocity parameter $\alpha$ and collisional broadening $\sigma$ (given in Bohr radius $a_{0}$ ) from Barklem et al. (2000).

The estimated reliability ranges for temperature and line-ofsight velocity of these lines are specified in Koza et al. (2006). The formation heights of the line cores and corresponding optical depths specified in Table 1 demonstrate the large extent of the height of the photospheric layers probed by the selected spectral lines. The reliability of the inversion also depends strongly on the accuracy of the atomic parameters. To avoid systematic errors in temperature and line-of-sight velocity $v_{\text {los }}$, we checked the accuracy of $\lambda_{\text {lab }}$ and oscillatory strengths $\log (g f)$ by a twocomponent model of the solar photosphere and by the method suggested in Borrero \& Bellot Rubio (2002). The advantage of this method is that the check on $\lambda_{\text {lab }}$ and $\log (g f)$ can be performed at the same time. Table 1 shows the $\log (g f)$ values with the best match of the synthetic and atlas profiles extracted from Neckel (1999). An exception was the Fe I 4994.1 A line, where the value for $\log (g f)$ adopted from Bard et al. (1991) did not need any modification. The check showed that the positions of the line centres of the synthetic profiles and the atlas profiles extracted from the FTS solar atlas agreed very well with only one exception: the laboratory central wavelength of the weak Fe I $4992.8 \AA$ line was decreased about $5 \mathrm{~m} \AA$. We used the classical Unsöld theory (Unsöld 1955) to compute the collisional broadening of Fe II $4993.3 \AA$ and Fe II $6456.4 \AA$ spectral lines. We checked the adequacy of the Unsöld theory synthesising the line profiles using the two-component model of the solar photosphere (Borrero \& Bellot Rubio 2002). The comparison of the synthetic profiles with the atlas profiles (Neckel 1999) showed a satisfactory match of the line wings without any need to increase the collisional broadening through an ad hoc enhancement (Bellot Rubio 1999). For the inversion we adopted the iron abundance $\log \epsilon_{\mathrm{Fe}} \mathrm{I}=7.46$ recommended in Asplund et al. (2000) and the calcium abundance $\log \epsilon_{\mathrm{Ca} \text { I }}=6.36$ given in Thévenin (1989).

\section{Results}

As a result of the inversion we obtained in total 47100 individual $1 \mathrm{D}$ models representing the space-time evolution of the physical parameters throughout the solar photosphere. We start the presentation of the results with a qualitative assessment of the final fits by the typical values of $\chi^{2}$ and equivalent signal-tonoise ratio eqS $/ N$. In the following we provide insight into the structure of the solar photosphere by the maps of the observed intensities and the inferred macroturbulent and line-of-sight 

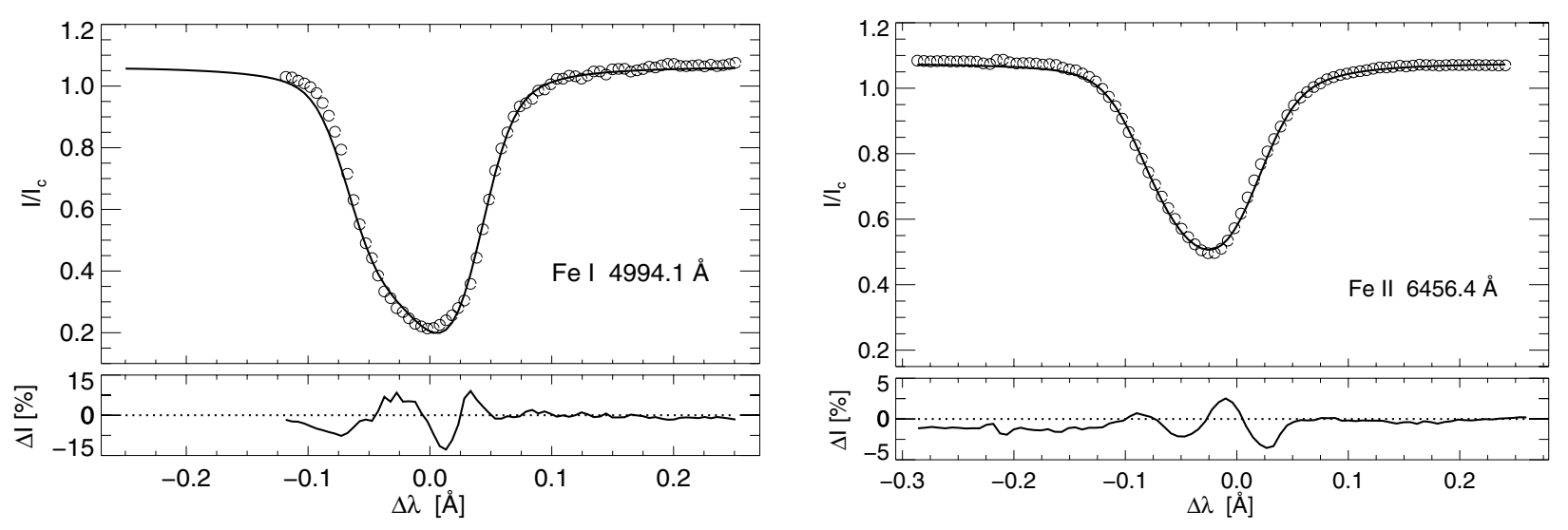

Fig. 3. Example of synthetic profiles (solid lines) resulting from the inversion and the observed profiles (circles) taken at 09:32:12 UT in the granule at $y=94^{\prime \prime}$ close to the upper border of the magnetic region. The lower panels show the relative residuals of the fits.

Table 2. Quality of the fits evaluated by typical equivalent signal-tonoise ratio $\langle e q S / N\rangle$ and typical value of $\left\langle\chi^{2}\right\rangle$ computed as time averages along the slit.

\begin{tabular}{llrr}
\hline \hline \multicolumn{2}{c}{ line $[\AA]$} & $\langle e q S / N\rangle$ & \multicolumn{1}{c}{$\left\langle\chi^{2}\right\rangle$} \\
\hline Fe I & 4992.8 & 113.4 & $9.8 \times 10^{-5}$ \\
Fe II & 4993.3 & 85.7 & $17.4 \times 10^{-5}$ \\
Fe I & 4994.1 & 68.9 & $28.2 \times 10^{-5}$ \\
Ca I & 6455.6 & 135.1 & $8.8 \times 10^{-5}$ \\
Fe II & 6456.4 & 114.3 & $11.0 \times 10^{-5}$ \\
\hline
\end{tabular}

velocities, and temperatures in selected optical depths. In order to compare them with some classical 1D models and the 3D numerical simulations we show the mean temperature and velocity stratifications, root-mean-square (rms) fluctuations, as well as an empirical relation between observed continuum intensity and line-of-sight velocity.

\subsection{Quality of inversion}

We plot an example of the observed and best-fit profiles resulting from the inversion in Fig. 3. The Doppler shifts and the asymmetrical shape of the profiles, which are the result of the velocity field present at the point of the observation, are reproduced to a reasonable degree of accuracy. Notice the highly asymmetric line core of the strong line Fe I 4994. 1 A.

The quality of the final fits resulting from the inversion is characterised by the values of $\chi^{2}$ and equivalent signal-to-noise ratio eqS $/ N$, which is defined following Borrero \& Bellot Rubio (2002) as the inverse of the standard deviation of the residuals. Table 2 summarises the typical values of eqS $/ N$ and $\chi^{2}$ of individual lines obtained by averaging along the slit and in time. For the examples in Fig. 3, the inversion yielded eqS $/ N=50$ and $\chi^{2}=45 \times 10^{-5}$ for the strong line Fe I 4994.1 $\AA$, while better values of $e q S / N=131$ and $\chi^{2}=9.5 \times 10^{-5}$ were obtained for the fit of the second line Fe II 6456.4 A. Generally, the best fits were achieved for the medium-strong line Ca I $6455.6 \AA$, while the fits of the strong line Fe I 4994.1 A exhibited the largest discrepancies.

Because response functions vary considerably both with optical depth and wavelength (e.g. Koza \& Kučera 2003), the uncertainties also change with optical depth and increase towards the limits of the sampled range. We estimated by the response functions for temperature and line-of-sight velocity
(Koza et al. 2006) that these spectral lines are sensitive to temperature and velocity changes within the ranges of the optical depths $\langle 0.8,-3.9\rangle$ and $\langle-0.2,-2.5\rangle$, respectively.

\subsection{The mean temperatures and line-of-sight velocities}

The horizontally and temporally averaged gas temperatures and line-of-sight velocities are shown in Fig. 4 separately for the non-magnetic and magnetic regions. Because we averaged more than $10^{4}$ values in each optical depth, the uncertainties are small so are not shown in the figures. All figures and results hereafter that are pertinent to the line-of-sight velocity keep the usual astrophysical sign convention, i.e. negative velocity indicates upflow, while positive suggests downflow.

The mean temperatures in the non-magnetic region is consistent with the classical 1D semiempirical models as well as with the mean of the 3D simulation by Stein \& Nordlund (1998). In the magnetic region the mean temperature stratification indicates that the sub-photospheric layers are cooler by about $100 \mathrm{~K}$ and the upper layers $\left(\log \tau_{5}<-3\right)$ are hotter by about $300 \mathrm{~K}$ than the $1 \mathrm{D}$ models and the 3D simulation. The velocities averaged in the non-magnetic region exhibit upflows over $\log \tau_{5} \in\langle-0.2,-2.6\rangle$ with the velocity decreasing toward the upper layers. In contrast, the 3D simulation by Stein \& Nordlund (1998) shows velocities of $\approx 0.1 \mathrm{~km} \mathrm{~s}^{-1}$ in these photospheric layers. In the upper layers of $\log \tau_{5}<-2.8$, both the observations and the 3D simulation show an almost zero mean velocity. In the magnetic region, the mean velocity exhibits wavy behaviour unlike both the nonmagnetic region and the $3 \mathrm{D}$ simulation.

\subsection{The photosphere in observations and inversion}

Figure 5 shows the space-time evolution of the observed linecentre intensities of four selected lines ordered with decreasing optical depth formation of the line cores. While the line center of the weak line Fe I $4992.8 \AA$ maps the lower photospheric layers at $\log \tau_{5}=-1.0$, the line core of the strong line Fe I $4994.1 \AA$ brings information about the low chromosphere at $\log \tau_{5}=-4.7$. These observations are supplemented by the map of continuum intensity shown in Fig. 6. The granulation is recognisable by white and dark strips in the non-magnetic region both in the continuum intensity map and in the low photospheric layers. In upper layers the oscillation pattern prevails in the linecentre intensity maps and dominates in the low chromosphere, as can be seen from the line-centre intensity of Fe I $4994.1 \AA$. 

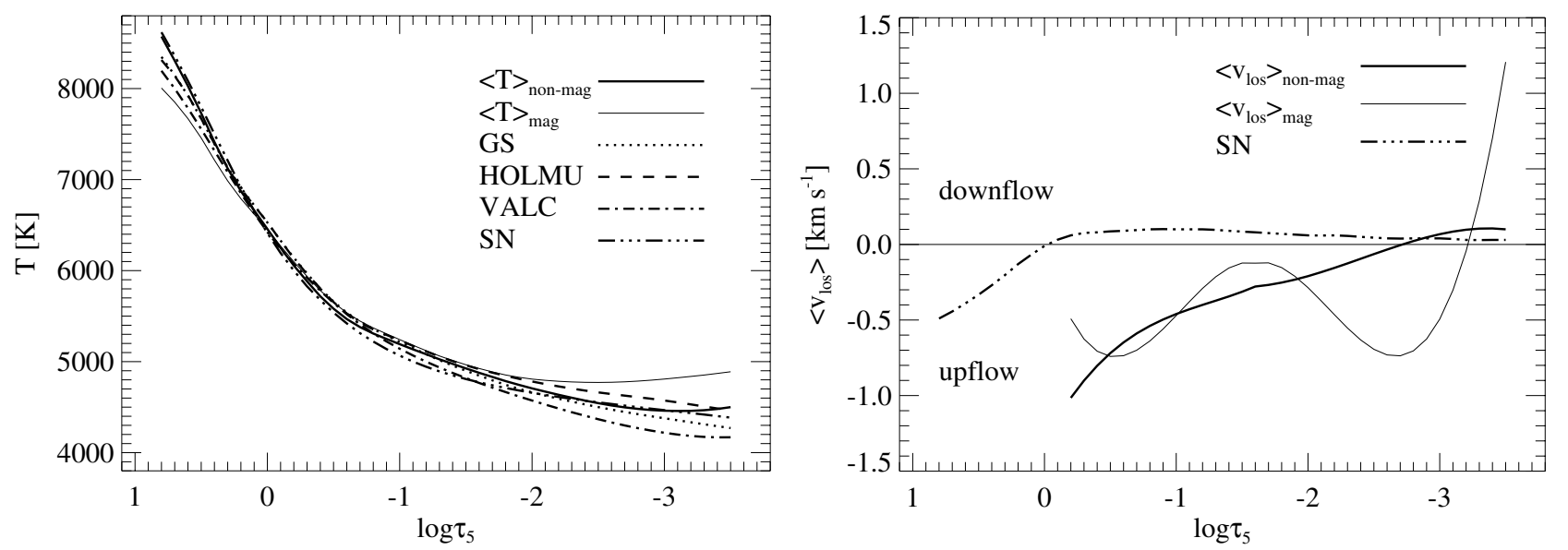

Fig. 4. Left: temperature stratifications. The mean in the non-magnetic $\langle T\rangle_{\text {non-mag }}$ and magnetic regions $\langle T\rangle_{\text {mag }}$ obtained by the inversion, the starting model GS (Grevesse \& Sauval 1999), the model atmospheres HOLMU by Holweger \& Müller (1974), VALC by Vernazza et al. (1981), and temporally and spatially averaged temperature from the 3D simulation SN by Stein \& Nordlund (1998). Right: the mean velocity in the nonmagnetic $\left\langle v_{\text {los }}\right\rangle_{\text {non-mag }}$ and magnetic $\left\langle v_{\text {los }}\right\rangle_{\text {mag }}$ regions. Temporally and spatially averaged vertical velocity SN is from the 3D simulation by Stein \& Nordlund (1998).

In these layers the magnetic region is identifiable by bright bands without any oscillatory pattern to indicate an increase of the linecentre intensities. An exception is the white band at $y=80^{\prime \prime}$ in the line-centre intensity map of the line Fe I 4994.1 $\AA$, which shows brightenings with an estimated period of $5 \mathrm{~min}$.

The structure of the solar photosphere resulting from the inversion is illustrated in Fig. 6 showing the layers where the continuum is formed and in Fig. 7 characterising the upper layers by maps of the macroturbulent velocity, temperature differences, and absolute velocities at the selected optical depths $\log \tau_{5}=$ $0.0,-0.2,-1.0,-1.2,-1.7$, and -2.5 out of the 49 layers considered in the inversion. The continuum intensity map and the map of temperature differences in Fig. 6 show that the observed intensity contrast of $0.8-1.1$ corresponds to temperature differences of 400-500 K between granules, intergranular lanes, and pores. Depending upon the time and the actual position with respect to the granular structure, the macroturbulent velocity $v_{\text {mac }}$ varies between $0.9 \mathrm{~km} \mathrm{~s}^{-1}$ and $1.9 \mathrm{~km} \mathrm{~s}^{-1}$, which is consistent with our results presented in Kučera et al. (2004). Detailed comparison of the continuum intensity map and $v_{\text {mac }}$ shows that in the non-magnetic region the lower values of $v_{\text {mac }}$ correspond to the granules, while $v_{\mathrm{mac}}$ in the intergranular lanes near to the upper limit of the interval. Owing to this, the map of $v_{\text {mac }}$ mirrors the continuum intensity map but on a reversed scale. The bright granule and the fragmenting granule at the positions $y=10^{\prime \prime}$ and $19^{\prime \prime}$ along the slit are good examples of this behaviour. This result is a manifestation of what has already been concluded by many previous, studies namely that the upflows appear laminar, while the downflows are more turbulent; i.e., they contain more unresolved velocity patterns. This picture was also supported by the numerical simulation by Stein \& Nordlund (1998). These characteristics generally hold in the magnetic region as well; however, the dark tracks resulting from the pore and the micropore at the positions $y=75^{\prime \prime}$ and $80^{\prime \prime}$ are co-spatial with the regions of increased $v_{\mathrm{mac}}$. The granular overshoot prevails on the velocity map at $\log \tau=-0.2$, and the strong upflows between $\approx-1.5$ and $-2 \mathrm{~km} \mathrm{~s}^{-1}$ are co-spatial with the fragmenting granule at $y=30^{\prime \prime}$ and the bright granules at $y=57^{\prime \prime}$ and $94^{\prime \prime}$. On the other hand, the intensive downdrafts of $1.7 \mathrm{~km} \mathrm{~s}^{-1}$ are observed in the vicinity of the pores at $y=75^{\prime \prime}$ and $80^{\prime \prime}$. Fast downflows on the borders of a pore and inside a bright point were found in the 3D MHD simulation by Stein \& Nordlund (2000, see
Fig. 18, p. 106). Granular fragmentations seen at $17^{\prime \prime}$ and $30^{\prime \prime}$ are recognisable in the continuum intensity map (Fig. 6) and the temperature maps (Fig. 7) throughout the lower photosphere up to the optical depth $\log \tau_{5}=-1.0$. This layer exhibits signatures of the temperature reversal apparent in the cases of both fragmenting granules, which are dark in contrast to their counterparts in the continuum. At the optical depth $\log \tau_{5}=-1.2$ and partly at $\log \tau_{5}=-1.7$, the temperature reversal can be still seen in the granules at $5^{\prime \prime}, 17^{\prime \prime}, 24^{\prime \prime}, 30^{\prime \prime}$, and $47^{\prime \prime}$. Positive correlation with continuum is re-established at $\log \tau_{5}=-2.5$ where the granules are again hotter than the surroundings, but now the granulation pattern is overlaid by 5-min oscillations. The temperature differences increase progressively with height, reaching maximum amplitude of $\approx 1300 \mathrm{~K}$ at the optical depth $\log \tau_{5}=-2.5$. In the middle photosphere the magnetic region can be identified easily by its track of temperature, which is about $300 \mathrm{~K}$ higher than in the non-magnetic region.

While the granular overshoot prevails in the velocity map around $\log \tau=-0.2$, the 5 -min oscillations dominate in the upper layers of the non-magnetic region. The patches of oscillation with coherent amplitude have an estimated size around 5"; however, their smooth pattern is perturbed in the magnetic region. Some exceptional events of granular evolution are also clearly seen in the velocity structure of the middle photosphere; see e.g. the fragmenting granule at $y=30^{\prime \prime}$ still visible at $\log \tau_{5}=-1.0$ and -1.2 . Even the upper photosphere at $\log \tau_{5}=-2.5$ still exhibits velocity structures induced by granulation as the upflows at $y=17,24$, and $47^{\prime \prime}$ demonstrate.

Most inversions yielded negligible microturbulence (not shown). In some cases the inversion finished with the values between $0.3 \mathrm{~km} \mathrm{~s}^{-1}$ and $0.9 \mathrm{~km} \mathrm{~s}^{-1}$ but without some clear and systematic relation to the granular structure in both regions.

\section{4. rms fluctuations in observations and simulations}

Figure 8 shows our observed rms fluctuations of temperature and line-of-sight velocity in the non-magnetic and magnetic regions. Following Wedemeyer et al. (2004), the fluctuations were computed as a square root of variance for the pertinent quantities using the mean stratifications inferred in the non-magnetic and 
J. Koza et al.: Photospheric modeling through spectral line inversion

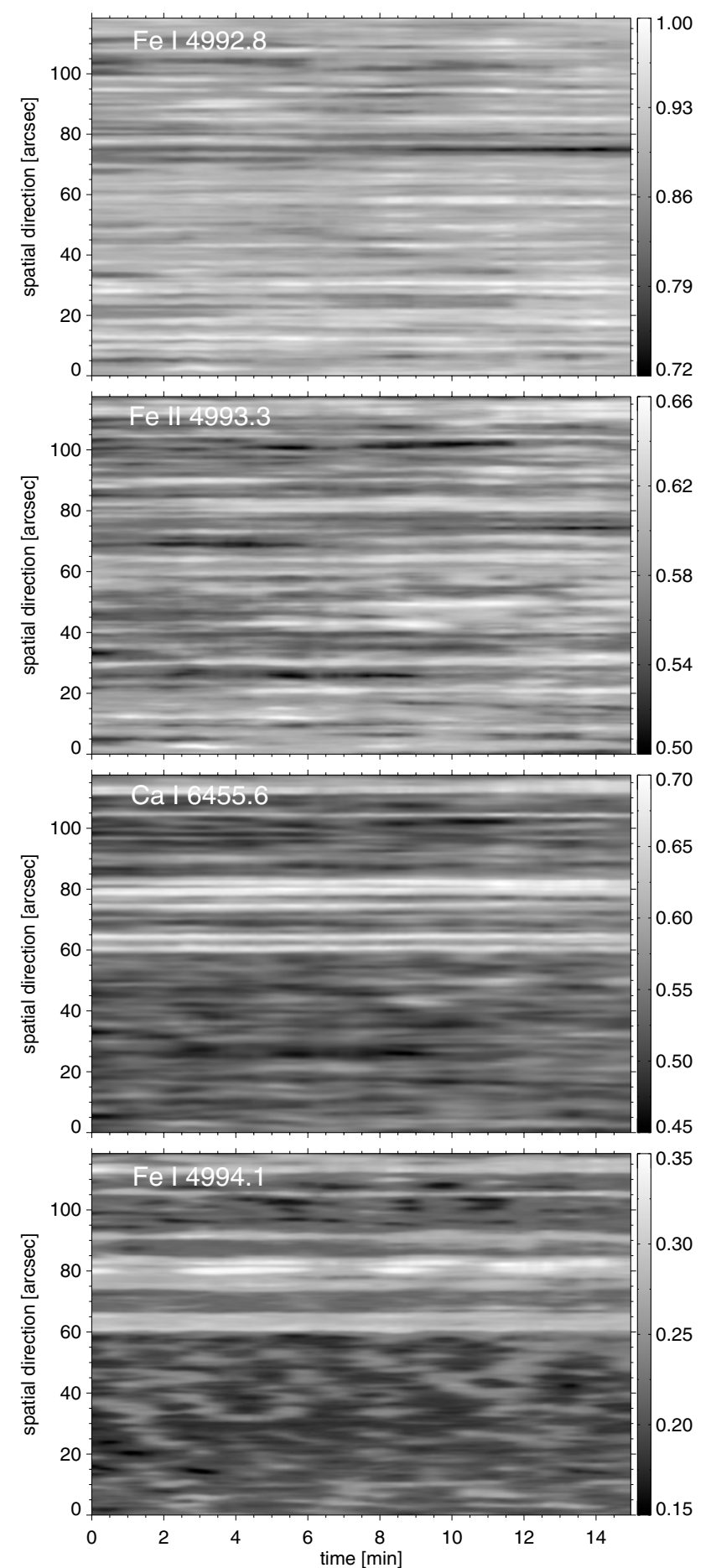

Fig. 5. Space-time evolution of the observed line-center intensities. The optical depth of formation of the line cores from top to bottom are: $\log \tau_{5}=-1.0,-1.2,-1.7$, and -4.7 . The greyscales are given as fractions of the continuum intensity.

magnetic regions (Fig. 4). Because the errors of the rms fluctuations are smaller than $1 \mathrm{~K}$ and $1 \mathrm{~m} \mathrm{~s}^{-1}$, they are not shown.

The sub-photospheric layers are characterised by a sharp drop in the rms temperature fluctuation with height both in the 3D simulations and the results inferred by the inversion, which are systematically lower at about $\approx 400 \mathrm{~K}$. Particularly, in the non-magnetic region they fall to $\approx 50 \mathrm{~K}$ at $\log \tau_{5}=0.0$, and higher they remain small up to $\log \tau_{5}=-2.0$. At larger heights the upper photosphere becomes more inhomogeneous. The

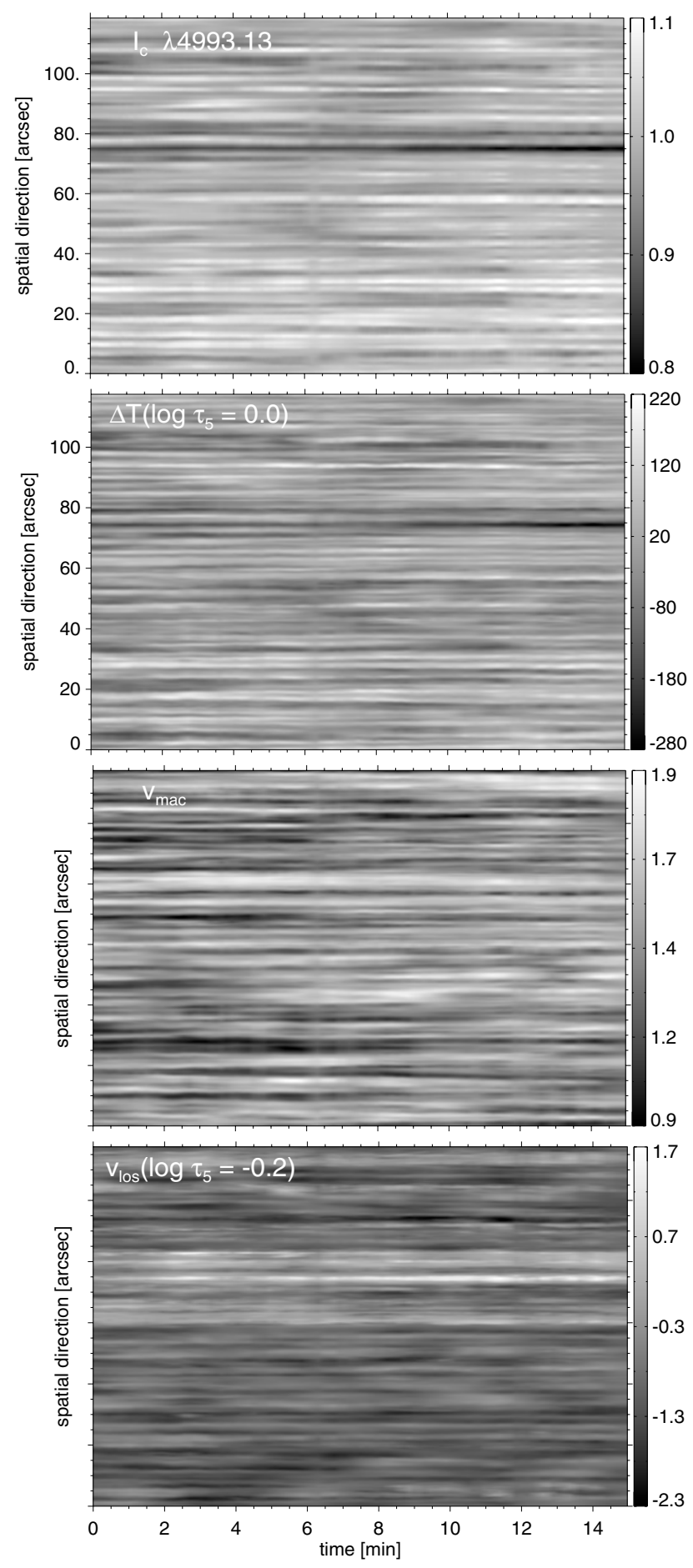

Fig. 6. Space-time evolution of continuum intensity, temperature differences at $\log \tau_{5}=0.0$ compared to the mean temperaturs in the nonmagnetic region, macroturbulent velocity, and line-of-sight velocities at $\log \tau_{5}=-0.2$, respectively from top to bottom. The greyscales are given as fractions of the continuum intensity, $\mathrm{K}, \mathrm{km} \mathrm{s}^{-1}$, and $\mathrm{km} \mathrm{s}^{-1}$, respectively. The gravitational redshift of $-636 \mathrm{~m} \mathrm{~s}^{-1}$ was subtracted from line-of-sight velocities. Negative velocities indicate upflows. The images were smoothed out by boxes of $3 \times 3$ pixels.

subsequent rise of the observed fluctuations up to $200 \mathrm{~K}$ over the $\log \tau_{5} \in\langle-2.0,-3.0\rangle$ range is followed by a rather steep increase in the upper photosphere. In the magnetic region, the rms temperature fluctuations behave similarly, but their values 

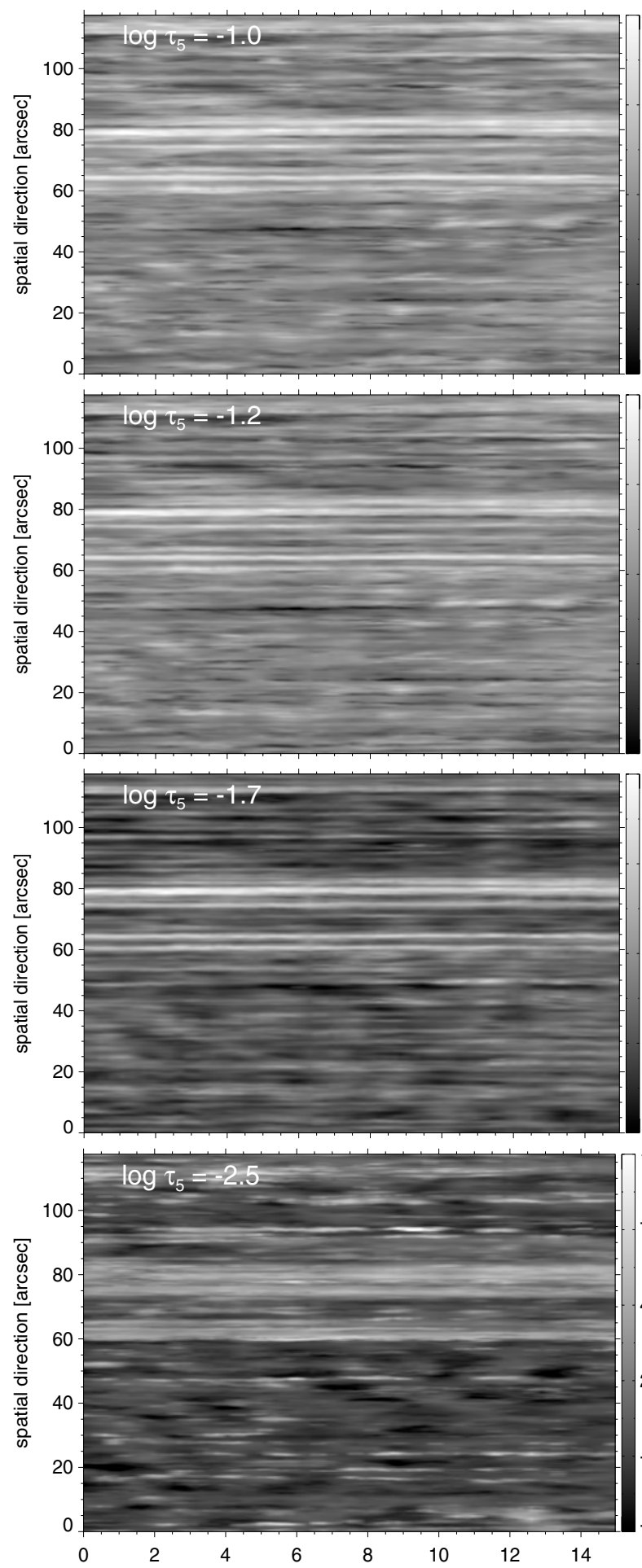
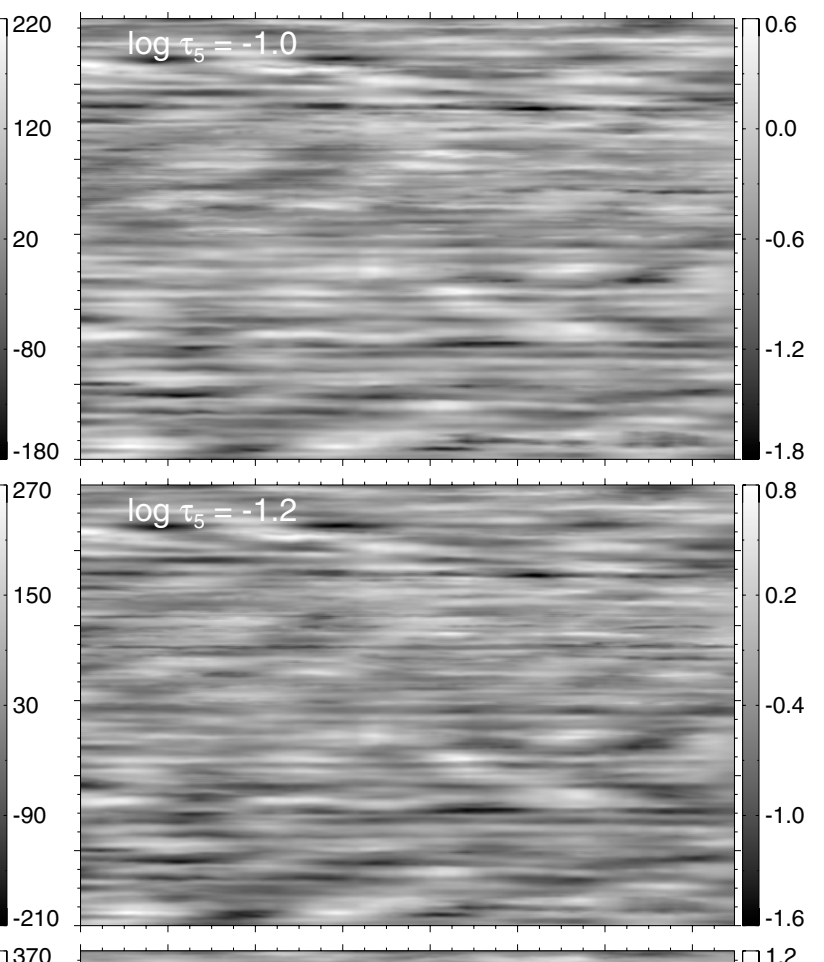

370

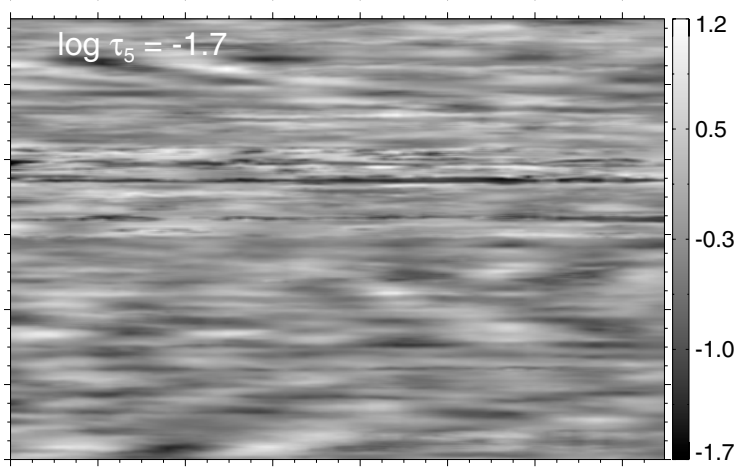

1000

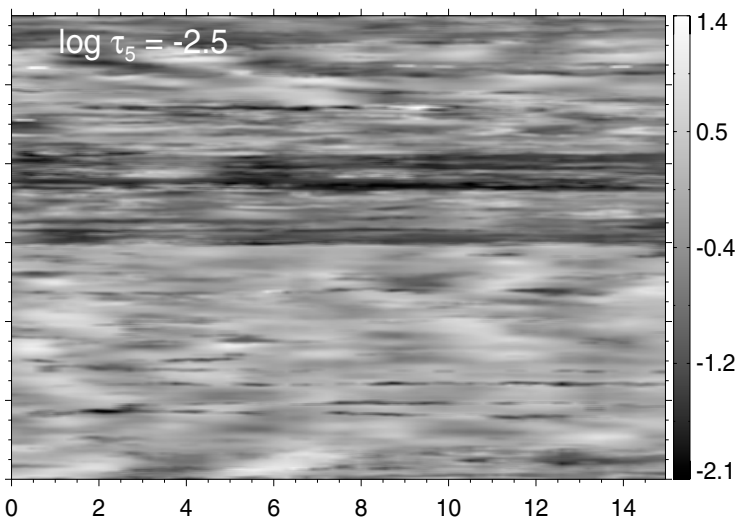

Fig. 7. Space-time evolution of temperature differences compared to the mean temperature stratification in the non-magnetic region $(l e f t)$ and lineof-sight velocities $(r i g h t)$ in the selected optical depths. The greyscales are expressed in Kelvin and $\mathrm{km} \mathrm{s}^{-1}$. The gravitational redshift of $-636 \mathrm{~m} \mathrm{~s}$ was subtracted from line-of-sight velocities. Negative velocities indicate upflows. The images were smoothed out by boxes of $3 \times 3$ pixels.

are higher and a slight rise up to $100 \mathrm{~K}$ is present over the low and the middle photosphere at $\log \tau_{5}>-2.0$.

In the same figure we also show the temperature fluctuations in the 3D granulation simulations of Wedemeyer et al. (2004, see p. 1131, Fig. 9) and Stein \& Nordlund (1998). In the low photosphere the 3D simulations exhibit a decrease in the rms temperature fluctuations with height reaching a minimum of $150 \mathrm{~K}$ at $\log \tau_{5}=-0.6$ and then increasing monotonically with height. Both 3D simulations behave rather similarly over $\log \tau_{5} \in\langle 0.8,-1.5\rangle$, but from $\log \tau_{5}=-1.5$ upwards the $3 \mathrm{D}$ simulation by Stein \& Nordlund (1998) exhibits nearly constant rms temperature fluctuations of about $200 \mathrm{~K}$ in discrepancy with our observational results, as well as, with the numerical results of Wedemeyer et al. (2004). Note the similarity of the gradients of the curves in the sub-photospheric layers and over interval $\log \tau_{5} \in\langle-2.0,-3.0\rangle$ in which our observed rms temperature 

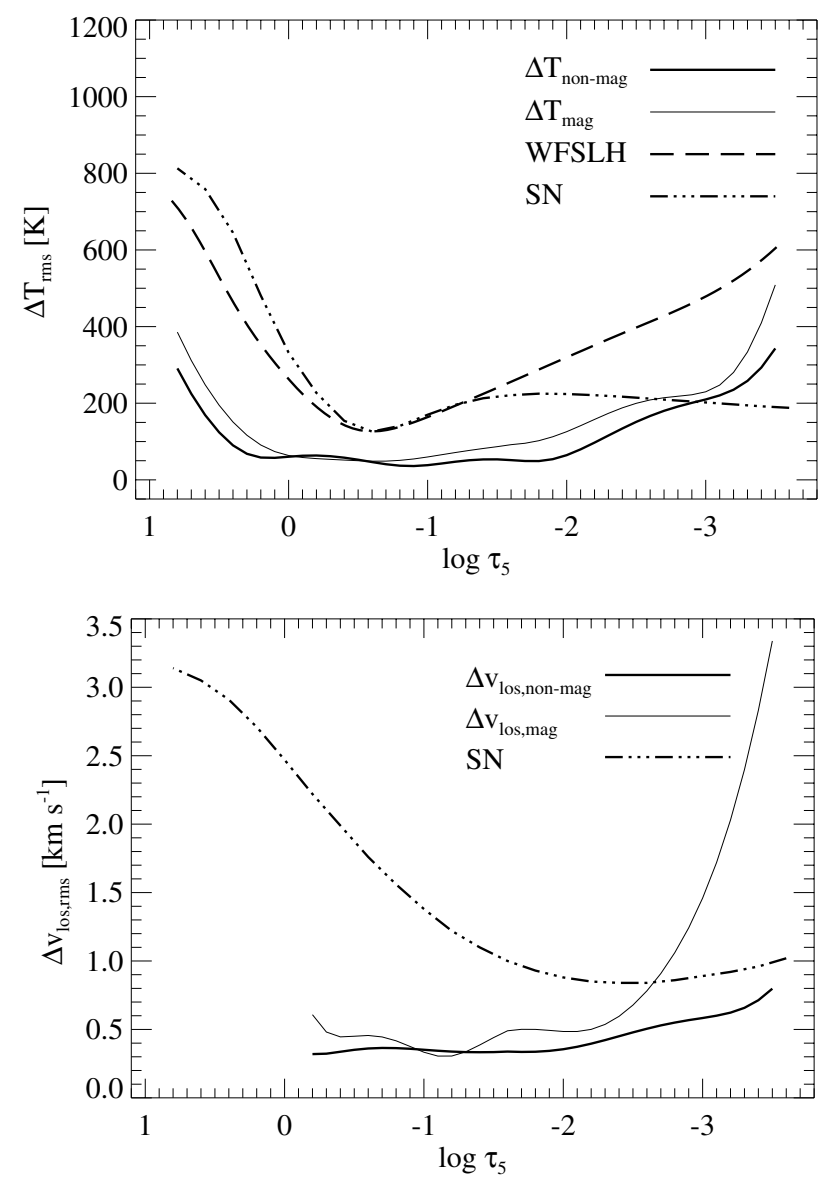

Fig. 8. Top: horizontally and temporally averaged rms temperature fluctuations in the non-magnetic $\Delta T_{\text {non-mag }}$ and magnetic $\Delta T_{\text {mag }}$ regions, as well as in the 3D numerical simulations WFSLH by Wedemeyer et al. (2004, see p. 1131, Fig. 9) and SN by Stein \& Nordlund (1998). Bottom: horizontally and temporally averaged rms line-of-sight velocity fluctuations in the non-magnetic $\Delta v_{\text {los,non-mag }}$ and magnetic $\Delta v_{\text {los,mag }}$ regions, as well as in the 3D numerical simulation SN by Stein \& Nordlund (1998).

fluctuations mimic the trend of the 3D simulation WFSLH by Wedemeyer et al. (2004).

Our observed velocity fluctuations in the non-magnetic region show the nearly constant value of $\approx 0.35 \mathrm{~km} \mathrm{~s}^{-1}$ over $\log \tau_{5} \in\langle-0.2,-2.0\rangle$, above which they rise up to $\approx 0.8 \mathrm{~km} \mathrm{~s}^{-1}$ at $\log \tau_{5}=-3.5$. The increase in velocity fluctuations is more pronounced in the magnetic region, reaching $3 \mathrm{~km} \mathrm{~s}^{-1}$ in the highest layers. In the same optical depths the 3D simulation by Stein \& Nordlund (1998) exhibits a decrease of rms velocity fluctuations from $2.22 \mathrm{~km} \mathrm{~s}^{-1}$ to $0.84 \mathrm{~km} \mathrm{~s}^{-1}$ followed by a slight increase to $1 \mathrm{~km} \mathrm{~s}^{-1}$ at $\log \tau_{5}=-3.5$.

\subsection{Empirical relations}

Figure 9 shows the empirical relation between observed continuum intensity and line-of-sight velocity at $\log \tau_{5}=-0.2$ inferred by inversion in the non-magnetic and magnetic regions, and outlines the estimated border of the area occupied by the results of the 3D numerical simulation by Stein \& Nordlund (1998, see p. 917, Fig. 3), who showed their simulated continuum intensity versus vertical velocity at the optical depth $\tau=1$. Obviously our observations and inversion give narrower ranges of continuum intensity and velocity than does the 3D numerical simulation. However, Stein \& Nordlund (1998) show these data without

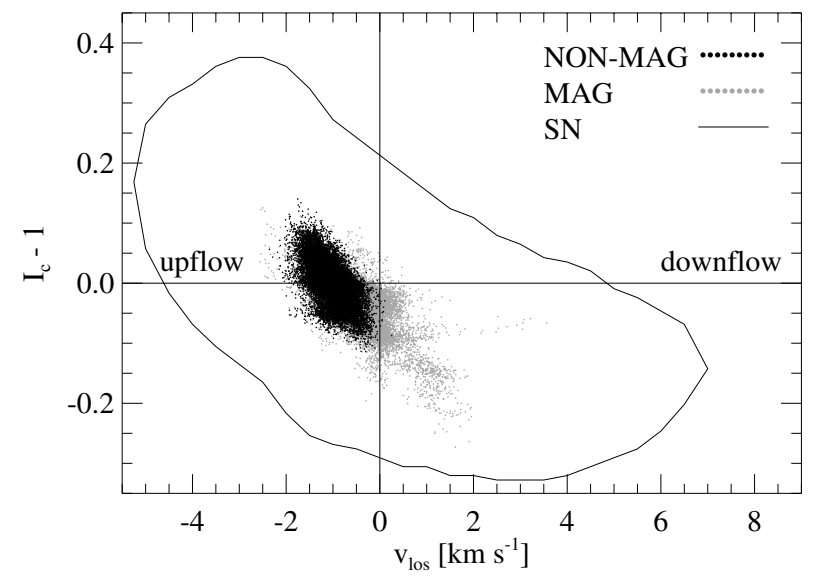

Fig. 9. Scatter plot of observed continuum intensity versus line-of-sight velocity inferred by the inversion at the optical depth $\log \tau_{5}=-0.2$ in the non-magnetic and magnetic regions. The thin solid line outlines the extents of these quantities obtained at the optical depth $\tau=1$ in the 3D numerical simulation SN by Stein \& Nordlund (1998, see p. 917, Fig. 3). Negative velocities indicate upflows, and the intensity axis shows relative continuum intensity reduced by unity.

smoothing by some point spread function. The centroid of our data is shifted about $\approx-1.0 \mathrm{~km} \mathrm{~s}^{-1}$ toward upflows. In this plot typical granules should be in the upper left quadrant and typical intergranular lanes in the lower right quadrant. However, both simulation and observations indicate unusual granular dynamics. A certain fraction of granules should exhibit downflows (Fig. 9, upper right quadrant) and in turn the upflows should occur in the abundant clump of intergranular lanes (Fig. 9, lower left quadrant). Thus the simulation predicts the occurrence of unresolved dark upflows and bright downflows associated with granular dynamics, which have not yet been observed and reported. The scatter ellipse of the non-magnetic region is asymmetric towards bright upflows, simply indicating that granules predominate over intergranular lanes in an averaged sample. Comparison of scatter plots from both regions shows that downflows occur much more frequently in magnetic than in the non-magnetic region.

\section{Discussion}

We applied the inversion code SIR on the sequence of the spectrograms with the typical signal-to-noise of about 140 and 640 in the spectral regions around $4993.8 \AA$ and and $6456.5 \AA$ and the quality of the resulting fits was summarised in Table 2. The largest departures in terms of the equivalent signal-to-noise $(e q S / N), \chi^{2}$ and the largest relative errors occurred in the case of the strong line Fe I 4994.1 A. Borrero \& Bellot Rubio (2002) inverted 22 medium-strong Fe I spectral line profiles extracted from the FTS solar atlas (Neckel 1999) with $S / N=2500$, and their inversion finished with the eqS / $N$ of about 211 . This quality evaluation suggests that further improvements in the fits are possible by the enhancement of the $S / N$ of the observations especially in the spectral region involving the strong lines.

From the deep investigations of Rutten (1988) and Shchukina \& Trujillo Bueno (2001), we know that almost all neutral iron lines show departures from LTE. A later paper concludes that it is possible to obtain good fits to the observed lines by assuming LTE with a suggested iron abundance of 7.46. Besides, the limited extent of the inversion just to the photospheric layers largely eliminates the influence of the NLTE effects, which become important in the lower chromosphere, 
i.e. at the optical depths $\log \tau_{5} \in\langle-4,-6\rangle$. Moreover, the NLTE effects can also be minimised using lines spanning a wide range of atomic parameters because each transition is influenced by the NLTE effects in different or even in opposite ways (Borrero \& Bellot Rubio 2002). Since we kept all these precautions as much in the selection of the iron abundance and the spectral lines as in the determination of upper boundary, we suppose that the NLTE effects do not exceed other uncertainties entering into the inversion much thereby influencing the results (e.g. instrumental profile of the spectrograph, atmospheric smearing, uncertainties in atomic parameters; etc.).

Also, ubiquitous magnetic fields play no less a role than the NLTE effects in formation of the spectral lines observed with high resolution. Since we lack the spectropolarimetric observations, the impact of the magnetic fields on our results can only be judged by the effective Landé factors $g_{\text {eff }}$ of selected spectral lines shown in Table 1. Generally, the analysed spectral lines exhibit low or medium sensitivity to the magnetic field. Nevertheless, we observed the solar photosphere by the spectral line with $g_{\text {eff }}=1.5$ and in the red portion of the solar spectrum keeping in mind that the Zeeman broadening depends upon the square power of the wavelength. Thus we can expect that the results characterising the pore and its surroundings are especially biased, as in the example of the high value of the macroturbulent velocity seen in Fig. 6 at the positions $y=75$ and $80^{\prime \prime}$ along the slit nearly where the pore and micropore are seen in the G-band filtergram (Fig. 1). Because there is no physical reason to expect the increased turbulence in the presence of the strong magnetic field, the inversion code interpreted the line profiles broadening in terms of higher values of macroturbulent velocity than usually obtained in the granules. Interestingly, the inversion finished with a zero microturbulent velocity in the same locations.

Applying the inversion code SIR to the spectral lines profiles emerging from the magnetic region, we found an increase in temperature compare to the external non-magnetic environment, which varies between 100 and $500 \mathrm{~K}$ (Fig. 8). Many authors reported on the enhanced internal temperature in the fluxtube models of solar plage (e.g. Solanki \& Stenflo 1985; Walton 1987; Bellot Rubio et al. 2000) and hotter atmosphere above a small pore (Sütterlin et al. 1996; Sütterlin 1998). Several factors were identified as potentially responsible for the increased internal temperature in the flux tubes. Usually the nearly evacuated interior of the flux tubes is taken as the main reason that the optical level $\tau=1$ in the flux tube is situated several tens of kilometers deeper than the same optical level in the surrounding non-magnetic photosphere that is an analogy of the Wilson depression. The scatter of hot-wall irradiance makes a spurious effect of the hot flux-tube interior. Keller et al. (2004) prove that the brightness of the solar faculae out of disk centre comes from irradiation of the limb-ward granules. But in the disk centre, a nudge from a nearby granule (Steiner et al. 1998) may bring the deeper and hotter flank of the granule into the line-of-sight of an observer through a nearly transparent flux-tube interior. The results of the 3D simulation (Stein \& Nordlund 1998) show a steep temperature gradient near the surface in the ascending flows that also determines the high contrast of the bright points. Bellot Rubio et al. (2000) propose that UV radiation emanating from the walls of the flux tubes causes overionization of the iron and leads to the weakening of the Fe I lines compared to their LTE strengths. Since all previous studies have treated the observations and the simulations in LTE, they interpreted the missing opacities in the line cores by increasing the internal temperature. Probably the most comprehensive account of increased temperature above a small pore and a hot flux-tube interior has
Table 3. Full width at half minimum differences $\triangle F W H M$ of synthetic profiles computed with constant $900 \mathrm{G}$ and zero field strength (synt), profiles observed in the pore and a granule (pore - gr) and in the pore, and an intergranular lane (pore - ig).

\begin{tabular}{|c|c|c|c|c|}
\hline \multirow[t]{2}{*}{ line } & \multirow[t]{2}{*}{$g_{\text {eff }} \lambda^{2}\left[10^{7} \AA^{2}\right]$} & \multicolumn{3}{|c|}{$\Delta F W H M[\mathrm{~m} \AA]$} \\
\hline & & synt & pore-gr & pore - ig \\
\hline Fe I 4992.8 & 3.2 & 4 & 4 & 4 \\
\hline Fe II 4993.3 & 1.5 & 3 & -1 & 15 \\
\hline Fe I 4994.1 & 3.7 & 5 & 30 & 28 \\
\hline Ca I 6455.6 & 4.2 & 9 & 22 & 30 \\
\hline Fe II 6456.4 & 5.0 & 11 & 29 & 35 \\
\hline
\end{tabular}

been achieved by the 3D MHD simulation by Stein \& Nordlund (2000, Fig. 18, p. 106).

To estimate whether $900 \mathrm{G}$ magnetic field alone can account for the broadening of spectral lines interpreted by the SIR code by increasing temperature and macroturbulent velocity in the pores at $y=75$ and $80^{\prime \prime}$, we performed the following numerical experiment along with measurements of the FWHM both of the synthetic and observed spectral line profiles (Table 3). With the model atmosphere of Grevesse \& Sauval (1999) we calculated the synthetic profiles of all lines, whose atomic parameters are shown in Table 1. Adding the magnetic field strength of $900 \mathrm{G}$ with zero value of inclination and azimuth to the model, we repeated this once again, thus simulating the influence of the magnetic field found in the pore. The resulting synthetic profiles were used as an input of the SIR code and were inverted under the same conditions as the observed ones. The resulting temperatures were about $100-150 \mathrm{~K}$ higher than the starting one at the optical depths $\log \tau_{5} \in\langle-1,-3\rangle$. Obviously this cannot fully account for the temperature enhancement found at the optical depths $\log \tau_{5}=-1.0,-1.2$, and -1.7 (Fig. 7) at the positions of $y=75$ and $80^{\prime \prime}$ corresponding to the pore and micropore. Furthermore in Table 3 we demonstrate the influence of $900 \mathrm{G}$ magnetic field on the $F W H M$ of the synthetic line profiles. Table 3 lists the $F W H M$ differences $(\triangle F W H M$ ) of synthetic lines profiles computed by $900 \mathrm{G}$ and zero field strengths as a function of the magnetic sensitivity of the spectral lines expressed by the factor $g_{\mathrm{eff}} \lambda^{2}$. To highlight the inability of this field strength to explain the observed spectral line broadening, we also show the $\triangle F W H M$ measured in the pore, the selected granule, and the intergranular lane.

For this purpose we chose the line profiles observed at one of the best seeing moments (Fig. 2) at $t=11.5 \mathrm{~min}$ and coming from the spatial points $y=75^{\prime \prime}, 31^{\prime \prime}$, and $24^{\prime \prime}$ corresponding to the pore, a granule, and an intergranular lane in the non-magnetic region (see the map of the continuum intensity in Fig. 6). Except for the weak spectral line Fe I $4992.8 \AA$ and Fe II $4993.3 \AA$ observed in the intergranular lane, all line profiles exhibited considerably larger broadening than one would expect from the adopted field strength. All these results suggest the presence of some additional broadening mechanism presented above pore and micropore. However, we used field strength derived from the MDI magnetogram, which is considerably lower than the typical values of a pore. Bumba (1967), Beckers \& Schröter (1968), Steshenko (1968), and Keppens \& Martinez Pillet (1996) give an average value of 1400-1700 G. An increase in temperature above a small pore with a diameter of $\approx 1.5^{\prime \prime}$ has already been found by the 3D MHD simulation by Stein \& Nordlund (2000). Also a small pore with a diameter of $2^{\prime \prime}$ analysed by Sütterlin et al. (1996) and Sütterlin (1998) exhibits over $\log \tau_{5} \in\langle-2,-4\rangle$ 
a temperature higher by about $400 \mathrm{~K}$ than the model atmosphere VALC (Vernazza et al. 1981) in the same layers.

Figure 8 shows that our observed temperature fluctuations are significantly smaller than the computed ones at all depths, with flatter height dependence over $\log \tau_{5} \in\langle 0.0,-2.0\rangle$. These disagreements are likely to be due to atmospheric smearing, limited spatial resolution of the telescope, instrumental scattered light, the instrumental profile of the spectrograph, and misfits between observed and synthetic line profiles resulting from imperfect inversion. The same accounts for the narrower ranges of continuum intensities and line-of-sight velocities in Fig. 9. In particular, the steep increase in the observed rms temperature fluctuation in the upper photosphere $(\log \tau<-3)$ in Fig. 8 is uncertain and may be due to acoustic modulation, which the SIR code interprets in terms of enhanced temperature variations. On the other hand, the simulations are likely to overestimate the rms temperature fluctuations in the upper photosphere through the wrong assumption on LTE.

The bright downflows and the dark upflows indicated by Fig. 9 have a physical as well as an observational origin. Approaching the photosphere, the buoyancy weakens and some hot parcels may begin to sink even before they have cooled down enough to become dark, so we observe them as bright downflows (Keller 2005). On the other hand, the turbulence causes certain recirculation of the cool matter back to the surface, which can be potentially identified by the dark upflows as proposed by Stein \& Nordlund (1998). Additionally, atmospheric smearing and instrumental limitations in observations are likely to contribute to their occurrence.

Finally, we suggest the plausible explanation for the nonzero mean velocity (Fig. 4) in the low and the middle photospheres and the offset of the centroid of velocities in the nonmagnetic region (Fig. 9) with an estimated position of about $\left(-1.0 \mathrm{~km} \mathrm{~s}^{-1}, 0.0\right)$. These features are likely to be due neither to a methodological error in the wavelength calibration nor to uncertainties of the wavelengths of the the reference spectral lines. We recall that the relative velocity of the spectrograph with respect to the disc centre was only $\approx 90 \mathrm{~m} \mathrm{~s}^{-1}$ and the wavelength uncertainties had a similar value. Nonetheless, a gravitational redshift of $636 \mathrm{~m} \mathrm{~s}^{-1}$ was subtracted from the resulting velocities. We consider that the predominance of the granules in the averaged sample, along with atmospheric smearing and instrumental limitations, are responsible for these features. The behaviour of the observed mean velocity in the non-magnetic region is also determined by the properties of the 5-min oscillations in different layers. Odert et al. (2005) show that, while the velocity autocorrelation function oscillates but keeps the sign for the spectral line formed in the low photosphere, for the spectral line formed in the upper photosphere, the function oscillates around zero, having a character of damped oscillations. This high regularity of the oscillatory pattern in the upper photosphere results in a zero observed mean velocity in the non-magnetic region at $\log \tau_{5}<-3.0$.

\section{Summary}

Using the time sequence of high-resolution spectrograms and the SIR inversion code, we inferred temporal evolution of the temperature, line-of-sight velocity, and macroturbulent velocity through the quiet solar photosphere and in a small area of enhanced magnetic activity. The most important aspects are our avoiding of spatial and temporal averaging and comparison of the results with the 3D numerical simulations of the solar granulation. According to our results, the lower photosphere is thermally homogeneous, exhibiting small rms temperature fluctuations, while the sub-photospheric and upper layers over $\log \tau_{5} \in\langle-2,-3\rangle$ show an increase in the fluctuations with similar gradient to the one in the 3D simulation by Wedemeyer et al. (2004). In contrast, the comparison with the 3D simulation by Stein \& Nordlund (1998) shows contradictory behaviour of the observed and predicted temperature fluctuations at $\log \tau_{5}<-2.0$. Our results generally exhibit reduced rms temperature fluctuations compared with the 3D models of Wedemeyer et al. (2004) and Stein \& Nordlund (1998) and narrower ranges of the observed continuum intensities and the velocities than the 3D simulation by Stein \& Nordlund (1998). We found a flat distribution of the rms velocity fluctuations in the low and middle photospheres over $\log \tau_{5} \in\langle-0.2,-2.5\rangle$, which are smaller than in the 3D simulation by Stein \& Nordlund (1998). The centroid of the observed continuum intensity-velocity distribution and the mean velocity in the low and middle photospheres are shifted toward upflows. We conclude that the predominance of the granules in the averaged sample, along with atmospheric smearing and instrumental limitations, causes these differences and the shifts. Our observations and the inversion confirm the existence of the region of enhanced temperature extended above the small pores found by Sütterlin et al. (1996) and Sütterlin (1998) and the dominance of the fast downflows in the magnetic region at the low photospheric layers at $\log \tau_{5}=-0.2$. These findings agree with predictions by the 3D MHD simulation by Stein \& Nordlund (2000). However, further study of this feature will have to be inevitably backed up by the spectropolarimetric observations.

Acknowledgements. The VTT is operated by the Kiepenheuer-Institut für Sonnenphysik, Freiburg, at the Observatorio del Teide of the Instituto de Astrofísica de Canarias. We are grateful to S. Wedemeyer-Böhm (KIS) for kindly providing the WFSLH data in Fig. 8. J.K. thanks M. Collados (IAC) for supervision and advice during his stay at the IAC in the early 2003 in the framework of Marie Curie Fellowship. The authors are grateful to R. J. Rutten for many comments. This work was supported by the Slovak grant agency VEGA $(2 / 6195 / 26)$ and by the Deutsche Forschungsgemeinschaft grant (DFG 436 SLK 113/7). This research is part of the European Solar Magnetism Network (EC/RTN contract HPRN-CT-2002-00313). J. Koza's research is supported by a Marie Curie Intra-European Fellowships within the 6th European Community Framework Programme.

\section{References}

Allende Prieto, C., \& García López, R. J. 1998a, A\&AS, 129, 41 Allende Prieto, C., \& García López, R. J. 1998b, A\&AS, 131, 431 Asplund, M., Nordlund, Å., Trampedach, R., \& Stein, R. F. 2000, A\&A, 359, 743

Asplund, M., Grevesse, N., Sauval, A. J., Allende Prieto, C., \& Kiselman, D. 2004, A\&A, 417, 751

Asplund, M., Grevesse, N., Sauval, A. J., Allende Prieto, C., \& Kiselman, D. 2005, A\&A, 435, 339

Bard, A., Kock, A., \& Kock, M. 1991, A\&A, 248, 315

Barklem, P. S., Piskunov, N., \& O’Mara, B. J. 2000, A\&AS, 142, 467

Beckers, J. M. 1969, A table of Zeeman Multiplets, Sacramento Peak Observatory Contribution No. 141 - Physical Science Research Papers No. 371 (Sacramento Peak Observatory and Air Force Cambridge Research Laboratories)

Beckers, J. M., \& Schröter, E. H. 1968, Sol. Phys., 4, 142

Bellot Rubio, L. R. 1999, A user guide to SIR, Version 2, Instituto de Astrofísica de Canarias

Bellot Rubio, L. R., Ruiz Cobo, B., \& Collados, M. 2000, ApJ, 535, 489

Borrero, J. M., \& Bellot Rubio, L. R. 2002, A\&A, 385, 1056

Bumba, V. 1967, Sol. Phys., 1, 371

Grevesse, N., \& Sauval, A. J. 1999, A\&A, 347, 348

Gurtovenko, E. A., \& Kostyk, R. I. 1989, Fraunhofer spectrum and a system of solar oscillator strengths (Naukova Dumka, Kiev)

Holweger, H., \& Müller, E. A. 1974, Sol. Phys., 39, 19 
Keller, C. U. 2005, private communication

Keller, C. U., Schüssler, M., Vögler, A., \& Zakharov, V. 2004, ApJ, 607, L59

Keppens, R., \& Martinez Pillet, V. 1996, A\&A, 316, 229

Koza, J., \& Kučera, A. 2003, Contrib. Astron. Obs. Skalnaté Pleso, 33, 224

Koza, J., Kučera, A., Rybák, J., \& Wöhl, H. 2006, in Proceedings of NSO Workshop \#23: Solar MHD: Theory and Observations - a High Spatial Resolution Perspective, ed. J. Leibacher, H. Uitenbroek, \& R. Stein, ASP Conf. Proc. Ser., 43

Kučera, A., Koza, J., Bellot Rubio, L. R., et al. 2004, Hvar Obs. Bull., 28, 19

Kupka, F., Piskunov, N. E., Ryabchikova, T. A., Stempels, H. C., \& Weiss, W. W. 1999, A\&AS, 138, 119, (VALD-2)

Moore, C. E. 1972, A multiplet table of astrophysical interest - Pt.1: Table of multiplets - Pt.2: Finding list of all lines in the table of multiplets, rev. edn. (NSRDS-NBS, Washington: US Department of Commerce)

Moore, C. E., Minnaert, M. G. J., \& Houtgast, J. 1966, The solar spectrum $2935 \AA$ to $8770 \AA$ (National Bureau of Standards Monograph, Washington: US Government Printing Office)

Nave, G., Johansson, S., Learner, R. C. M., Thorne, A. P., \& Brault, J. W. 1994, ApJS, 94, 221

Neckel, H. 1999, Sol. Phys., 184, 421

Odert, P., Hanslmeier, A., Rybák, J., Kučera, A., \& Wöhl, H. 2005, A\&A, 444, 257

Press, W. H., Teukolsky, S. A., Vetterling, W. T., \& Flannery, B. P. 1992, Numerical recipes in FORTRAN. The art of scientific computing, 2nd edn. (Cambridge: University Press)

Puschmann, K., Vázquez, M., Bonet, J. A., Ruiz Cobo, B., \& Hanslmeier, A. 2003, A\&A, 408, 363

Puschmann, K. G., Ruiz Cobo, B., Vázquez, M., Bonet, J. A., \& Hanslmeier, A. 2005, A\&A, 441, 1157
Rimmele, T., Dunn, R., Richards, K., \& Radick, R. 1999, in Proc. High Resolution Solar Physics: Theory, Observations, and Techniques, ed. T. R. Rimmele, K. S. Balasubramaniam, \& R. R. Radick, ASP Conf. Ser., 183, 222 Ruiz Cobo, B., \& del Toro Iniesta, J. C. 1992, ApJ, 398, 375

Rutten, R. J. 1988, in ASSL Vol. 138: IAU Coll. 94: Physics of Formation of FE II Lines Outside LTE, 185

Rybák, J., Wöhl, H., Kučera, A., Hanslmeier, A., \& Steiner, O. 2004, A\&A, 420, 1141

Schröter, E. H., Soltau, D., \& Wiehr, E. 1985, Vistas in Astronomy, 28, 519

Shchukina, N., \& Trujillo Bueno, J. 2001, ApJ, 550, 970

Sheminova, V. A. 1998, A\&A, 329, 721

Solanki, S. K., \& Stenflo, J. O. 1985, A\&A, 148, 123

Stein, R. F., \& Nordlund, A. 1998, ApJ, 499, 914

Stein, R. F., \& Nordlund, Å. 2000, Sol. Phys., 192, 91

Steiner, O., Grossmann-Doerth, U., Knoelker, M., \& Schuessler, M. 1998, ApJ, 495, 468

Steshenko, N. V. 1968, in Structure and Development of Solar Active Regions, ed. K. O. Kiepenheuer, IAU Symp., 35, 201

Sütterlin, P. 1998, A\&A, 333, 305

Sütterlin, P., Schröter, E. H., \& Muglach, K. 1996, Sol. Phys., 164, 311

Thévenin, F. 1989, A\&AS, 77, 137

Unsöld, A. 1955, Physik der Sternatmosphären, Zweite Auflage (SpringerVerlag)

Vernazza, J. E., Avrett, E. H., \& Loeser, R. 1981, ApJS, 45, 635

Vögler, A., Shelyag, S., Schüssler, M., et al. 2005, A\&A, 429, 335

Walton, S. R. 1987, ApJ, 312, 909

Wedemeyer, S., Freytag, B., Steffen, M., Ludwig, H.-G., \& Holweger, H. 2004, A\&A, 414, 1121

Wöhl, H., Kučera, A., Rybák, J., \& Hanslmeier, A. 2002, A\&A, 394, 1077 